\title{
Silk Fibroin Pads for Whole Blood Glucose Determination ${ }^{+}$
}

\author{
Augusto Márquez 1,*, Salvador D. Aznar-Cervantes ${ }^{2}$, J. L. Cenis ${ }^{2}$, Carlos Domínguez ${ }^{1}$ and \\ Xavier Muñoz-Berbel ${ }^{1}$ \\ 1 Institut de Microelectrònica de Barcelona, (IMB-CNM, CSIC), 08193 Bellaterra (Barcelona), Spain; \\ carlos.dominguez@imb-cnm.csic.es (C.D.); xavier.munoz@imb-cnm.csic.es (X.M.-B.) \\ 2 Instituto Murciano de Investigación y Desarrollo Agrario y Alimentario (IMIDA), \\ 30150 La Alberca (Murcia), Spain; sdac1@um.es (S.D.A.C.); josel.cenis@carm.es (J.L.C.) \\ * Correspondence: augusto.marquez@imb-cnm.csic.es; Tel.: +34-935-94-77-00 (ext. 2433) \\ + Presented at the Eurosensors 2018 Conference, Graz, Austria, 9-12September 2018.
}

Published: 30 November 2018

\begin{abstract}
In this work, we present a durable and miniaturized photonic lab on a chip (PhLOC) integrating functionalized silk fibroin pads for optical glucose quantification in whole blood samples. The PhLOC consists of a poly(methyl methacrylate) (PMMA) disposable structure for the pad holding and a coupling system for commercial optic fibers connection. The silk matrix, together with the PMMA design, has the capability to separate the plasma from the cell fraction of whole blood by plasma diffusion into the silk, minimizing the absorbance interferences from the hemoglobin of the erythrocytes. Then, the enzymes present in the matrix react with the glucose in the sample and produce a color change in an amplified process where the analyte-mediatormatrix interacts sequentially, increasing the common signal from the oxidized mediator ( 2 fold). The sensor works in the adequate linear range to distinguish between healthy and unhealthy glucose levels ( 0 to $12 \mathrm{mM}$ ), with a sensitivity of 0.084 a.u. $\mathrm{mM}^{-1}$, a limit of detection of $0.18 \mathrm{mM}$ and a limit of quantification of $1.44 \mathrm{mM}$. Furthermore, durability of the sensor is remarkable, maintaining its response unchangeable during the first 7 months of tests.
\end{abstract}

Keywords: optical biosensor; silk fibroin; durable biosensor; enzyme matrix; green chemistry

\section{Introduction}

The field of commercial point of care tests for glucose monitoring in blood is widely covered with the glucose test strips for electrochemical measuring. Colorimetric analysis in this case is poor developed, while in other tests ( $\mathrm{pH}$, pregnancy, lead acetate) is commonly used. This aspect is logic as blood has a strong red color due to the presence of hemoglobin, what suppose an important interference. To prevent this, cellulose is commonly employed to filter the sample, but also to manage the fluidics and retain the interactive elements (i.e., lateral flow tests). In this work, we replace cellulose with silk fibroin to provide, besides capillary pumping, and blood filtering, the possibility to immobilize enzymes and mediator inside the matrix and measure optically through it due to its high transparency.

\section{Materials and Methods}

For the extraction and purification of the silk fibroin [1], cocoons of Bombyx mori, obtained from silkworms reared in the sericulture facilities of the IMIDA (Murcia, Spain), were chopped in 4 or 5 pieces and boiled in $0.02 \mathrm{M} \mathrm{Na}_{2} \mathrm{CO}_{3}$ for 30 min to remove the glue-like sericin proteins. Then, raw fibroin was rinsed thoroughly with water and dried at room temperature for 3 days. The extracted 
fibroin was dissolved in 9.3 M LiBr (Sigma Aldrich, Saint Louis, MO, USA) for $3 \mathrm{~h}$ at $60^{\circ} \mathrm{C}$ to generate a $20 \% w / v$ solution that was dialyzed against distilled water for 3 days (Snakeskin Dialysis Tubing $3.5 \mathrm{kDa}$ MWCO, Thermo Scientific, Waltham, MA, USA) with eight total water exchanges. The resulting $7-8 \% w / v$ fibroin solution was stored at $4{ }^{\circ} \mathrm{C}$ until the preparation of films.

The functionalized silk fibroin films were prepared dissolving different amounts of glucose oxidase (GOx), horseradish peroxidase (HRP) and 2,2'-azino-bis(3-ethylbenzothiazoline-6-sulphonic acid) (ABTS) (all from Sigma Aldrich (Saint Louis, MO, USA)) in the 7\% $w / v$ aqueous silk fibroin solution and then cast into Petri dishes of $51 \mathrm{~mm}$. The silk fibroin was dried and exposed to $0.8 \mathrm{bar}$ atmosphere with high humidity during $12 \mathrm{~h}$ to obtain water annealed thin films of $10 \mu \mathrm{m}$.

The PMMA sample carrier was constructed layer-by-layer cutting $0.5 \mathrm{~mm}$ PMMA layers (Good Fellow) by $\mathrm{CO}_{2}$ laser ablation (Epilog mini 24) and joining them with pressure sensitive adhesive (PSA) sheets. The functionalized silk fibroin layer was cut in $4 \mathrm{~mm}$ diameter circular pads and introduced in the carrier.

For the measurements, $4 \mu \mathrm{L}$ of sample were charged in the PhLOC while the silk fibroin pads got wet by capillary pumping of the liquid from the sample. The subsequent color change was registered with a QE Pro Spectrometer (Ocean Optics) using a UV-Vis Deuterium-Halogen Lamp (Ocean Optics) as light source.

\section{Results and Discussion}

\subsection{Analyte Detection and Secondary Reaction}

The final deep purple color measured at $545 \mathrm{~nm}$ is given by a three steps reaction that involves two enzymes and also the silk fibroin matrix (Figure 1). First, the glucose is oxidized to gluconolactone by the GOx, generating $\mathrm{H}_{2} \mathrm{O}_{2}$ as a byproduct. The HRP reduces then the $\mathrm{H}_{2} \mathrm{O}_{2}$ to $\mathrm{H}_{2} \mathrm{O}$ while oxidizes ABTS, generating a green intermediate (ABTS radical) which is commonly used as the final colorimetric signal. The ABTS radical absorbance spectrum presents four absorbance bans in the visible: one centered at $410 \mathrm{~nm}$ and a broad band with three ovelaped contributions at 630, 745 and $820 \mathrm{~nm}$. However, this radical demonstrates capability to react with the tyrosine residues [2] of the silk fibroin, changing completely the absorbance spectrum: all the contributions in the visible disapear and an unique band centered at $545 \mathrm{~nm}$ finally remains (Figure 2). This last contribution presents the double of absorbance intensity respect to the first initial signal.

$$
\begin{aligned}
& \text { B-D-glucose }+\mathrm{O}_{2} \stackrel{\text { Glucose Oxidase }}{\longrightarrow} \text { D-glucono-1,5-lactone }+\mathrm{H}_{2} \mathrm{O}_{2} \\
& \mathrm{H}_{2} \mathrm{O}_{2}+\text { ABTS } \stackrel{\text { Horseradish Peroxidase }}{\longrightarrow} \mathrm{H}_{2} \mathrm{O}+\text { ABTS }^{+} \\
& \text {ABTS }^{+\cdot}+\text { Tyrosine } \longrightarrow \text { ABTS-Tyrosine bond }
\end{aligned}
$$

Figure 1. Scheme of the cascade reaction followed by the detection of glucose by reading the final product, ABTS radical bonded to the silk fibroin matrix. 


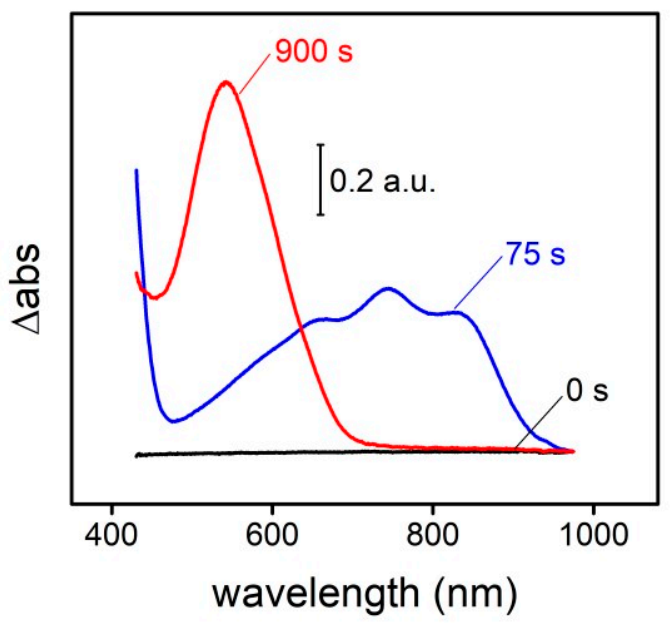

Figure 2. Functionalized silk fibroin absorbance spectra at different times ( 0,75 and $900 \mathrm{~s})$ after contact a PBS solution containing $12 \mathrm{mM}$ glucose.

\subsection{Biosensor Optimization}

To obtain an optimum response of the silk fibroin, three ABTS quantities ( 3,6 and $9 \% \mathrm{wt})$ were crossed with four GOx quantities (0.5, 1, 2 and 3\% wt) maintaning the HRP always in a 3:2 w/w relation respect to the GOx. The obtained results indicate that a good sensitivity and linearity are obtained using enzymatic quantities from 1 to 3\% wt. However, the amount of ABTS influenced the satruration response (Table 1), being the maximum quantity ( $3 \% \mathrm{wt}$ ) which gave the highest value. Nevertheless, for the final biosensor, the conditions of $6 \%$ wt of ABTS and 2\% wt of GOx were chosen as they offer a good compromise between the quatity of enzymes and mediator needed and the response.

Table 1. Different mediator quantity conditions and response in terms of sensitivity and linearity of the biosensor in case of $2 \%$ wt of GOx in the final silk fibroin matrix.

\begin{tabular}{cccc}
\hline GOx (\% wt)ABTS (\% wt)Sensitivity (abs (a.u.) [gluc] (mM)-1) Linearity $\left(\mathbf{R}^{2}\right)$ \\
\hline 2 & 3 & 0.04675 & 0.85837 \\
2 & 6 & 0.089 & 0.99406 \\
2 & 9 & 0.09425 & 0.99994 \\
\hline
\end{tabular}

\subsection{Biosensor Durability}

In terms of durability, the response of the silk fibroin was monitored in during 7 months without any apararent loss of sensitivity. To follow the sensitivity, three glucose conditions $(0,4$ and $8 \mathrm{mM})$ were measured for triplicate, wetting the pads the pads and transport by diffusion with PBS with those contents of analyte and registering the absorbance at $545 \mathrm{~nm}$.

\subsection{Whole Blood Analysis}

For whole blood analysis, the well constructed in the PMMA structure permitted to the blood liquid fraction of the $4 \mu \mathrm{L}$ blood sample to wet the functionalized silk fibroin pad, diminishing the interference that would introduce the haemoglobin present in the cell fraction of the samples (Figure 3). The glucose present in the spiked blood samples $(4,8$ and $12 \mathrm{mM})$ provoked a proportional color change registered by an absorbance increment at $545 \mathrm{~nm}$. 

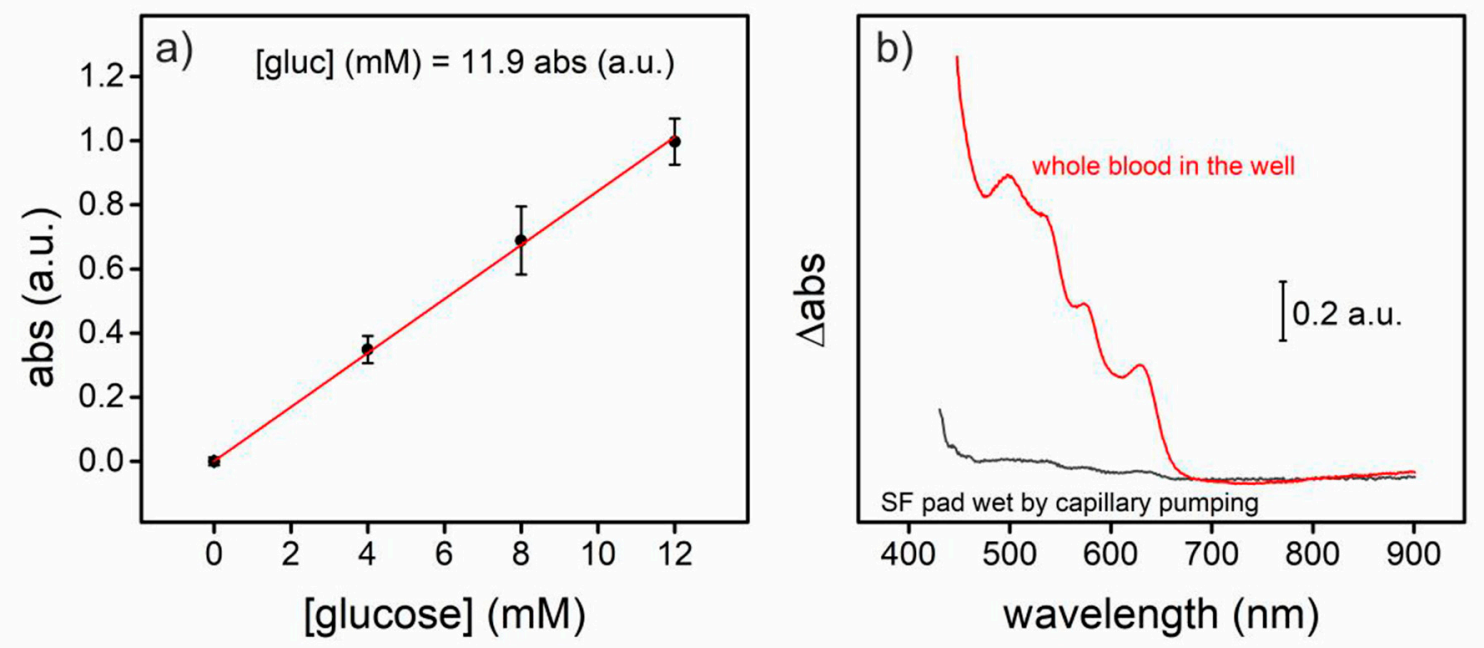

Figure 3. (a) Calibration curve of glucose spiked whole blood samples using the presented PhLOC. (b) Importance of the capillary pumping of the whole blood by the silk pads in terms of optical interferences of haemoglobin absorbance peaks.

\section{Conclusions}

In conclusion, a PhLOC is presented, capable of analyse whole blood samples highly removing optical interferences of whole blood by capillary pumping of the liquid fraction of the samples. The sensor presents high durability (7 months) besides good sensitivity $\left(11.9 \mathrm{mM}\right.$ abs.units $\left.{ }^{-1}\right)$, good limit of detection $(0.18 \mathrm{mM})$ and a sufficient limit of quantification $(1.44 \mathrm{mM})$.

Author Contributions: A.M., C.D. and X.M.-B. conceived, designed the experiments, analyzed the data and wrote the paper; A.M. performed the experiments; S.D.A.C. and J.L.C. contributed reagents/materials/analysis tools.

Acknowledgments: The authors acknowledge financial support of the ERDF/FEDER Operative Program of the Region of Murcia (Project No. 14-20-01) and Spanish R \& D National Program (MEC Project TEC2014-54449-C31-R). Dr. S.D. Aznar-Cervantes acknowledges the financial support of his research contract, program INIACCAA (DOC INIA 2015), announced by the National Institute for Agricultural and Food Research and Technology (INIA) and supported by The Spanish State Research Agency (AEI) under the Spanish Ministry of Economy, Industry and Competitiveness. Dr. X. Muñoz-Berbel also wants to acknowledge the "Ramón y Cajal" program from the Spanish Government. MSc. A. Márquez is also grateful to the MICINN for the award of a research studentship from FPI program.

Conflicts of Interest: The authors declare no conflict of interest.

\section{References}

1. Rockwood, D.N.; Preda, R.C.; Yücel, T.; Wang, X.; Lovett, M.L.; Kaplan, D.L. Materials fabrication from Bombyx mori silk fibroin. Nat. Protoc. 2011, 6, 1612-1631, doi:10.1038/nprot.2011.379.

2. Åkerström, B.; Maghzal, G.J.; Winterbourn, C.C.; Kettle, A.J. The lipocalin $\alpha_{1}$-microglobulin has radical scavenging activity. J. Biol. Chem. 2007, 282, 31493-31503, doi:10.1074/jbc.M702624200.

(C) 2018 by the authors; Licensee MDPI, Basel, Switzerland. This article is an open access article distributed under the terms and conditions of the Creative Commons Attribution (CC BY) license (http://creativecommons.org/licenses/by/4.0/). 